\title{
Glottopol
}

Revue de sociolinguistique en ligne

$33 \mid 2020$

Varia - Liberté, égalité, diversité !

\section{Hommages à Jean-Baptiste Marcellesi}

Nicolas Sorba et Philippe Blanchet

\section{(2) OpenEdition}

Journals

Édition électronique

URL : https://journals.openedition.org/glottopol/516

DOI : 10.4000/glottopol.516

ISSN : 1769-7425

Éditeur

Presses universitaires de Rouen et du Havre

Référence électronique

Nicolas Sorba et Philippe Blanchet, « Hommages à Jean-Baptiste Marcellesi », Glottopol [En ligne], 33 | 2020, mis en ligne le 01 janvier 2020, consulté le 02 octobre 2021. URL : http://

journals.openedition.org/glottopol/516; DOI : https://doi.org/10.4000/glottopol.516

Glottopol 


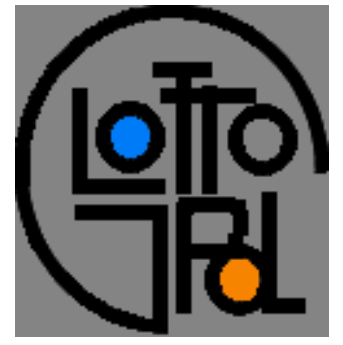

\section{GLOTTOPOL}

Revue de sociolinguistique en ligne n`33 - février 2020

Varia : Liberté, égalité, diversité !

\section{SOMMAIRE}

Hommages à Jean-Baptiste Marcellesi : À ringrazià vi, par Niculau Sorba ; "Ciao Ziu ! » par Philippe Blanchet.

Clara Mortamet : Présentation du numéro : Liberté, égalité, diversité !

Manon Him-Aquilli : "Prendre la parole sans prendre le pouvoir ». La mise en registre de l'horizontalité dans les assemblées générales anarchistes/autonomes.

Siham Hocini : La formulation du désaccord dans les débats télévisés algériens : quels "effetsde-politesse "?

Pauline Rannou: Trajectoires de la surdité en France. Parents entendants d'enfants sourds: questionner la fragmentation de l'identité sourde.

Catherine Combaz-Champlaine: Les rectifications orthographiques de 1990 comme révélateurs du rapport des enseignants à l'orthographe.

Marie-Madeleine Bertucci : Éléments d'épistémologie pour l'étude des espaces ségrégués des banlieues urbaines sensibles de la région parisienne.

Élise Gandon: Usages du numérique et illectronisme: nouvelles tâches pour le lecteurscripteur, nouveaux enjeux d'apprentissage.

Marc Arabyan : Quelle langue pour la recherche neurolinguistique française?

José R. de Arellano : Le rapprochement entre l'espagnol et le portugais pendant la décennie dorée sud-américaine : une problématique de l'horizontalité supercentrale.

Adam Wilson: Normes interactionnelles globalisées et communautés de pratique discontinues : les dynamiques sociolinguistiques du tourisme international.

Mélanie Lancien : Caractérisation de la variation liée à la situation de communication : apport de l'acoustique à la phonostylistique.

Assémou Maurice Ludovic Assémou : La prononciation du français parlé en Côte d'Ivoire.

http://glottopol.univ-rouen.fr 


\section{HOMMAGES À JEAN-BAPTISTE MARCELLESI}

\section{À ringrazià vi}

A porta di u sapè era sempri aparta sbalasgiata, incù quidda sapienza sviluppata da i radichi chì francò mari è monti. À passà l'anni à ascultà la ghjenti pà capiscia ciò ch'iddi diciani è ciò ch'iddi vuliani, pà ridà li dopu a so virità, suventi nigata è sbagliata da a sucità soia.

Incù i vosci travadda di ricerca, a suciolinguistica corsa hà trovu a so fonti è s'hè busca un maestru. A via presa da a lingua corsa, dapo l'annati 1980, hè stata guidata da sti cuncetti è nuzioni chì ani custruttu a voscia nomina. I Corsi l'ani fatti soii, appiichendu li. I minurità linguistichi si pudarani arrimbà pà u sempri à nantu à st'elaburazioni : individuazioni, ricunniscenza/nascita, satellisazioni, gluttopulitica... è pulinumia. St'ultimu cuncettu, par mè singulari, n'avemu parlatu mondi duranti i nosci scambii è scontri. Dà a priurità à u populu, à a pluralità, à a dimucrazia era a voscia primura è sti valori vosci si ritrovani tutti in u cuncettu di lingui pulinomichi. Pugnemu, incù i me culleghi, à trasmetta stu campu epistemulogicu riccu à l’Università di a voscia Corsica.

Mai ùn sminticaraghju st'usciu apartu. Mai ùn sminticaraghju sta vulintà trimenda di sparta a cunniscenza. Sempri sareti un asempiu di mudistia è generusità. Sempri sareti un mudeddu di circadori attori. Pà u dì, u fà è u da fà insignatu, O Ghjuvan Battì, à ringrazià vi.

Niculau Sorba, Università di Corsica

\section{«Ciao Ziu ! ». Témoignage de respect, de gratitude et d'affection à Jean- Baptiste Marcellesi}

Jean-Baptiste Marcellesi est considéré par beaucoup, par moi y compris, comme l'un des «pères fondateurs » de la sociolinguistique en France. La publication avec B. Gardin de la célèbre Introduction à la sociolinguistique : la linguistique sociale en 1974, puis l'organisation du premier grand colloque de sociolinguistique à Rouen en 1980 (dont les actes sont publiés en 1981), en constituent les fondations. Dès le départ, d'ailleurs, il avait pour la sociolinguistique une ambition vaste et transversale, puisqu'il n'a jamais séparé la sociolinguistique comme cadre théorique de beaucoup de questions linguistiques et apparentées, ce dont témoignent ses nombreuses publications, souvent cosignées : analyse de discours notamment politiques (dite « à entrée lexicale »), didactique du français, lexicologie, néologie... C'est de Jean-Baptiste que j'ai appris, très tôt, que la sociolinguistique n'est pas un complément à la linguistique, mais une autre linguistique qui répond aux limites de la linguistique classique, à sa « crise ». 
À partir des années 1980, la centration non exclusive de ses travaux sur les langues dites " régionales " et notamment sur le corse a pu laisser penser que son travail d'élaboration théorique (toujours collectif) y était étroitement limité. Je pense que c'est une erreur de perspective. Bien sûr les grandes enquêtes menées avec ses collègues de Corte, l'élaboration d'une reconnaissance du corse comme langue et non comme variété(s) de l'italien (du toscan, au nord) ou du sarde (au sud), la mise en place d'une glottopolitique polynomique, ont eu comme ancrage principal le terrain corse. Et ce n'est pas la moindre des réussites que d'avoir été l'un des acteurs principaux qui a donné son énergie et son savoir au riacquistu de la langue populaire de son ile, au point dont on observe les nombreux résultats, positifs à mon sens, aujourd'hui. Mais les concepts qu'il élabore, en sociolinguistique, prioritairement à partir de ce terrain, s'organisent en un cadre théorique global qui est radicalement sociolinguistique. Il s'agit d'intégrer l'ensemble du champ linguistique dans une théorie sociale et non de proposer un complément social à une linguistique structurale ou interne. Sa sociolinguistique n'est pas complémentaire à une linguistique; elle s'inscrit grandement en contradiction d'une linguistique non sociale (comme l'est aussi la sociolinguistique de L.-J. Calvet, autre "père fondateur »). En ce sens, ce cadre théorique est à la fois global et transversal.

D'autres ont pu se l'approprier et en bénéficier tout en l'adaptant à d'autres situations (socio)linguistiques, souvent de langues «minoritaires » (je l'ai fait pour le provençal). Mais cette théorie n'est pas exclusivement réservée aux situations minoritaires, y compris parce que Jean-Baptiste Marcellesi était un homme ouvert et généreux, toujours intéressé par d'autres terrains, d'autres combats, toujours prêt à partager ses savoirs avec d'autres, avec de jeunes chercheur·e·s. En 1998, quand j'ai publié ma traduction en provençal de Alice's Adventures in Wonderland de Lewis Carroll, sous le titre Li venturo de Liseto au païs estraourdinàri, l'éditeur m'avait organisé une tournée de conférence pour présenter mon livre dans les grandes villes de Provence. Jean-Baptiste l'a su et m'a invité à venir le présenter à Nans, au Cercle des Travailleurs.

J'ai été très tôt un lecteur assidu, abonné, aux Cahiers de Linguistique Sociale, à partir de 1982 je crois, et j'ai racheté tous les numéros parus. J'ai soutenu mon DEA en 1984, entamant aussitôt une thèse de $3^{\mathrm{e}}$ cycle à forte dimension sociolinguistique, et cette lecture m'ouvrait des perspectives nouvelles et enthousiasmantes. Thierry Bulot m'a raconté que, lorsque le labo de Rouen a décidé d'arrêter cette revue en 1996, la première lettre de protestation (amicale) qui leur était parvenue était la mienne. Et quand Jean-Baptiste est venu à Rennes pour l'entretien que nous avons filmé en 2003, il nous a confié, devant la caméra, avoir perdu le ${ }^{\circ} 1$ des CLS, consacré à « La Norme ». Je l'avais et je lui ai rapporté dès le lendemain (après en avoir fait une copie), ce volume rouge format A4 dactylographié que je lui ai offert. Et puis, lors d'une de mes dernières visites à Jean-Baptiste, à Nans en Provence où il s'était retiré pour la santé de son épouse Christiane, il est allé chercher dans son bureau le petit livret publié par le PC corse dont il était l'auteur (Pour une politique démocratique de la langue, 1985), dont il m'a offert un exemplaire original que je conserve précieusement. Il était très heureux d'avoir pu convaincre le PC, dont l'orientation était plutôt centraliste, de changer de point de vue sur les langues dites régionales. Je garde de ces échanges de publications, parmi nos discussions, un souvenir empli de l'affection, de la gratitude et du respect que je lui porte.

Jean-Baptiste Marcellesi était aussi italophone, suivant de près le développement de la sociolinguistique en Italie, notamment autour de T. de Mauro. Il a très tôt introduit dans sa sociolinguistique un certain nombre de concepts empruntés à la philosophie politique d'A. Gramsci, notamment celui d'hégémonie, dont le pouvoir explicatif s'est révélé puissant. Mais aussi celui de locuteur collectif, inspiré de l'intellectuel collectif de Gramsci. Car Jean-Baptiste Marcellesi a toujours puisé dans une inspiration marxiste, hétérodoxe au sens où, comme Gramsci et Bourdieu, il accorde une importance fondamentale aux éléments linguistiques, culturels, symboliques, dans la construction des rapports sociaux, notamment aux rapports de 
domination qu'il n'a eu de cesse de mettre à jour, de dénoncer et de combattre, et pas seulement en Corse (son travail en Normandie, en Kabylie, au Vietnam, en ex-RDA, en témoigne). Ma pratique familiale et universitaire de l'italien, mon attachement à mes origines italiennes, mon intérêt pour Gramsci grâce à un professeur d'italien au lycée qui nous faisait étudier ses Lettres de prison, m'avaient préparé à accueillir, là aussi avec enthousiasme, les apports gramsciens à la sociolinguistique que proposait Jean-Baptiste.

Mais Jean-Baptiste Marcellesi était un homme modeste, trop modeste, quoique déterminé dans ses convictions et dans les moyens d'actions scientifiques, institutionnels, syndicaux et politiques. Il n'a jamais réuni l'ensemble de sa pensée ni l'ensemble de ses travaux les plus significatifs en un volume monographique ou même collectif qui aurait fait date, par exemple en fin de carrière. Il aura fallu que T. Bulot, formé à Rouen et moi-même, lecteur assidu, qui considérons Jean-Baptiste Marcellesi comme un maitre à penser, nous les réunissions en un volume paru en 2003. La préparation de ce volume est passée par un long entretien filmé à Rennes, disponible sur la webTV de l'université Rennes 2 et transcrit dans ce même volume, qui constitue je crois une archive essentielle pour comprendre l'œuvre globale de Jean-Baptiste Marcellesi. Il nous a fait l'honneur de ce partage après nous avoir fait celui de son amitié.

Plusieurs des héritiers de sa pensée et de son action travaillent, en Corse et ailleurs, à faire mieux connaitre l'importance de cette pensée. Plusieurs volumes d'hommage viendront bientôt s'ajouter à ceux déjà publiés dont celui que certain'e $\cdot s$ de ses collègues lui ont consacré pour son départ en éméritat (Covariations pour un sociolinguiste, 1998). Un volume, en préparation bien avant son décès, paraitra au printemps 2020 comme numéro de la principale revue anglophone de sociolinguistique, l'International Journal of the Sociology of Language. J'y ai rédigé en anglais le texte d'ouverture, qui présente ce que je pense être un appareil théorique global et ce que je crois être sa puissance heuristique. Une version en français d'à peu près le même texte avec les mêmes objectifs constitue le premier chapitre de mes Éléments de sociolinguistique générale parus en 2018.

C'est dire tout ce que je dois à Jean-Baptiste Marcellesi.

Avec Thierry Bulot, nous disions que notre unité de recherche à Rennes 2 portait, à la suite de l'école de corso-rouennaise, une sociolinguistique radicale. J'ajoutais même souvent: « canal historique », en clin d'œil facétieux mais sincère à Jean-Baptiste et à nos collègues corses avec qui nous continuons à travailler étroitement. Canal historique, parce que je me sens héritier intellectuel de Jean-Baptiste Marcellesi, dont les échanges amicaux avec moi me sont restés comme autant de legs. Thierry Bulot n'est plus à mes côtés pour partager cet héritage dont il était inspiré davantage encore que je peux l'être. Il nous reste à faire vivre sa pensée scientifique importante, son engagement sociolinguistique et ses qualités humaines.

En Méditerranée, nous appelons « tonton » en français, « l'ouncle » en provençal, « ziu » en corse, «Amou » en algérien, un homme de la génération de nos parents ou grands-parents pour qui nous avons du respect et de l'affection.

« Ciao Ziu ! Adessias l’Ouncle ! Ma salam Amou ! ». Tu n’es pas vraiment parti. Tu restes auprès de nous.

Philippe Blanchet

PREFICS - Université Rennes 2 


\section{GLOTTOPOL}

Revue de sociolinguistique en ligne

Comité de rédaction : Michaël Abecassis, Salih Akin, Sophie Babault, Claude Caitucoli, Véronique Castellotti, Régine Delamotte, Robert Fournier, Stéphanie Galligani, Emmanuelle Huver, Normand Labrie, Foued Laroussi, Benoit Leblanc, Fabienne Leconte, Gudrun Ledegen, Danièle Moore, Clara Mortamet, Alioune Ndao, Isabelle Pierozak, Gisèle Prignitz.

Rédactrice en chef : Clara Mortamet.

Comité scientifique : Claudine Bavoux, Michel Beniamino, Jacqueline Billiez, Philippe Blanchet, Pierre Bouchard, Ahmed Boukous, Pierre Dumont, Jean-Michel Eloy, Françoise Gadet, Monica Heller, Caroline Juilliard, Jean-Marie Klinkenberg, Jean Le Du, Marinette Matthey, Jacques Maurais, Marie-Louise Moreau, Robert Nicolaï, Didier de Robillard, Paul Siblot, Claude Truchot, Daniel Véronique.

\section{Comité de lecture pour ce numéro :}

Mickael Abecassis, Michelle Auzanneau, Salih Akin, Céline Amourette, Gabriel Bergounioux, Philippe Boula de Mareuil, Catherine Brissaud, Aude Bretegnier, Maria Candea, Véronique Castellotti, Régine Delamotte, Jean-François De Pietro, Catherine Delarue-Breton, Anne Dister, Alexandre Duchêne, Valentin Feussi, Françoise Gadet, Stéphanie Galligani, François Gaudin, Monica Heller, Emmanuelle Huver, Caroline Juilliard, Abou Bakri Kebe, Jean-Marie Klinkenberg, Christian Lagarde, Foued Laroussi, Fabienne Leconte, Evelyne Lloze, Nolwenn Lorenzi, Gudrun Ledegen, Nadja Maillard, Marinette Matthey, Bruno Maurer, Véronique Miguel Addisu, Saskia Mugnier, Claire Oger, Marielle Rispail, Richard Sabria, Véronique Traverso, Christel Troncy, Cécile Van den Avenne. 